\title{
Kawasaki disease and soy: potential role for isoflavone interaction with Fcy receptors
}

\author{
Michael A. Portman ${ }^{1,2}$
}

Kawasaki disease (KD) is a diffuse vasculitis occurring in children and showing predilection for the coronary arteries. The etiology remains unknown, although some risk factors for susceptibility have been defined. Asian ethnicity is a primary risk factor. Several theories have circulated regarding the differences in KD ethnic incidence. Those theories implicating genetic differences among populations as the cause for this discrepancy have dominated and are areas of active investigation by multiple research groups. Differences in diet between Asians and Westerners are touted as reasons for certain ethnic-related discrepancies in susceptibility to cardiovascular disease and cancer in adults. Surprisingly, these cultural dietary differences have not been previously considered as the source of the discrepancy in KD incidence among these ethnic populations. Recent data from genetic studies have highlighted the role of specific immune receptors in the pathogenesis of KD. Functions of the Fc $\gamma$ receptors (FcGRs) are modulated by isoflavones in soy, in particular, genistein. Epidemiological data from Hawaiian populations support an association between soy consumption and KD. These observations form the basis of a hypothesis: isoflavones participate in KD pathogenesis by modulating function of the FCGRs and by disrupting the balance between activation and inhibition of the inflammatory response.

K awasaki disease (KD) is a diffuse vasculitis occurring in children. This disease causes inflammation with coronary artery dilation and aneurysm formation. Described by Tomisaku Kawasaki initially in 1967, KD now represents the leading cause of acquired heart disease in developed nations (1). The etiology remains unknown, although some of the factors defining susceptibility have been identified. Asian ethnicity provides the primary risk. KD incidence in Japan exceeds 200/100,000 children, $>10$ times the rate in the United States (2). Eastern Asian countries including Korea and Taiwan (3) also show remarkably high KD incidence as compared with countries with populations of predominantly Caucasian or European descent (4). The high incidence persists in American children of Japanese descent (5). Several theories have circulated regarding these ethnic differences in KD incidence. Those implicating genetic differences among populations as the defining factor predominate (3). Genetic studies have identified differences in human leukocyte antigen and CD40 loci, which might account for some but not all of the ethnic diversity in KD incidence (6). Although population density and climatic factors have been associated with $\mathrm{KD}$ outbreaks, these do not explain ethnic differences in $\mathrm{KD}$ incidence that persist throughout the world (3). Ethnic and cultural disparities in diet have not been previously considered as potential causes for the variation in KD incidence. Soy consumption in Asian cultures is substantially higher than that in the United States and Europe. Observational studies suggest that the Asian diet, which includes greater amounts of soy protein than do typical diets of non-Asian persons in the United States and Europe, may contribute to lower risk of cardiovascular disease and several cancers. These observations prompted the US Food and Drug Administration in 1999 to approve a health claim stating that diets that are low in saturated fat and cholesterol and include soy protein reduce the risk of heart disease (7). Following this, detailed evaluation by the Nutrition Committee of the American Heart Association in 2006 showed that more recent epidemiological studies and clinical trials did not support these assertions $(8,9)$. In addition, significant concerns have been raised regarding the potential adverse health effects of isoflavones such as those contained in soy, particularly in infants (10). Most of these concerns have stemmed from the capacity of the isoflavone genistein to bind to the estrogen receptor. Recent data from genetic studies have indicated a major role for Fc $\gamma$ receptors (FcGRs) in KD pathogenesis (6,11-13). Genistein has also been shown to inhibit FcGR function (14). The integration of these observations forms the basis of a hypothesis: isoflavones participate in $\mathrm{KD}$ pathogenesis by modulating the function of FcGRs and disrupting the balance between activation and inhibition of the inflammatory response. Furthermore, differences in isoflavone consumption through dietary ingestion of soy protein may explain the ethnic discrepancies in $\mathrm{KD}$ incidence.

\section{THE FcGRs AND KD}

Participation of the FcGRs in KD pathogenesis and susceptibility, as well as the potential impact of isoflavones on their functions, forms the central foundation of the hypothesis. The FcGRs are transmembrane receptors present on the surface of multiple types of immune cells. The extracellular domains of these receptors bind to the Fc portion of immunoglobulin $G$ 


\section{Kawasaki disease and isoflavones}

(IgG) antibodies present in immune complexes and play an important role in mediating the host defense against pathogens and in determining self-recognition and tolerance (reviewed by Nimmerjahn and Ravetch (15)). Genetic studies show that functional polymorphisms in these receptors alter susceptibility to autoimmune diseases such as systemic lupus (16).

These receptors are classified according to their binding affinities with IgG and are further classified according to their principal influence on inflammatory responses as activating or inhibiting (17). The FcGRs crosslink with each other (Figure 1), and the stoichiometries between the activating and inhibitory receptors that are involved determine the inflammatory responses by immune cells (15). High-dose intravenous immunoglobulin (IVIG) provides the principal therapy for $\mathrm{KD}$, presumably through interaction of IgG with the FcGRs (18). Mice lacking FcGR2B, the sole inhibitory FcGR, do not respond to IVIG, demonstrating the importance of this receptor to the anti-inflammatory response $(18,19)$. FcGR2B surface expression on monocytes and macrophages is upregulated in mice by binding of sialylated IgG (constitutes $~ 20 \%$ of human IgG) to non-integrin-related-1 (SIGN-R1) or the transgenically expressed human ortholog (DC-SIGN) $(18,19)$.

Single-nucleotide polymorphisms in the human $F c G R$ genes can modify transcriptional regulation and/or function of the encoded receptors, thereby altering either receptor expression and activity, or interaction among multiple receptors (20). IVIG resistance or refractoriness occurs in 15-20\% of patients with $\mathrm{KD}$, and these patients carry a relatively high risk for coronary

\section{Integrated Mechanism Review}

dilation and aneurysm (4). In the case of FcGR2B, singlenucleotide substitutions at $-120 \mathrm{~T} / \mathrm{a}$ and $-386 \mathrm{G} / \mathrm{c}$ enhance the binding of specific transcription factors, resulting in elevated FcGR2B expression on lymphocytes and monocytes (20). Recently, Shrestha et al. showed that Caucasian patients with KD harboring these single-nucleotide polymorphisms and their haplotypes, including both the alleles in tight linkage disequilibrium, showed substantially greater odds for therapeutic IVIG response than did patients with $\mathrm{KD}$ with the major alleles. Of note, these two single-nucleotide polymorphisms and their associated haplotype occur either rarely or not at all in Asians (13). Codominant alleles or haplotypes that modify functions of the activating FcGR2A and 3B also show significant associations for KD risk and IVIG treatment response, respectively, thereby creating potential population-based impacts. Three recent investigations using different methodologies have confirmed increased risk of acquiring $\mathrm{KD}$ in individuals harboring histidine in the FcGR2A-131H/R (rs1801274) polymorphism. Shrestha et al. (12), evaluating KD patients and their parents (trios) with transmission disequilibrium testing, showed that the FcGR2A-131H variant substantially increased the KD risk or susceptibility (odds ratio: 1.4-2.75) to KD. Khor et al. (11) identified the same locus as a risk factor in European and Asian (Korea, Taiwan, Hong Kong, Shanghai) populations in a large genome-wide association study with a case-control design. Onouchi et al. (6) replicated this genome-wide association study finding in a Japanese KD population. The increased risk associated with this polymorphism does not appear to

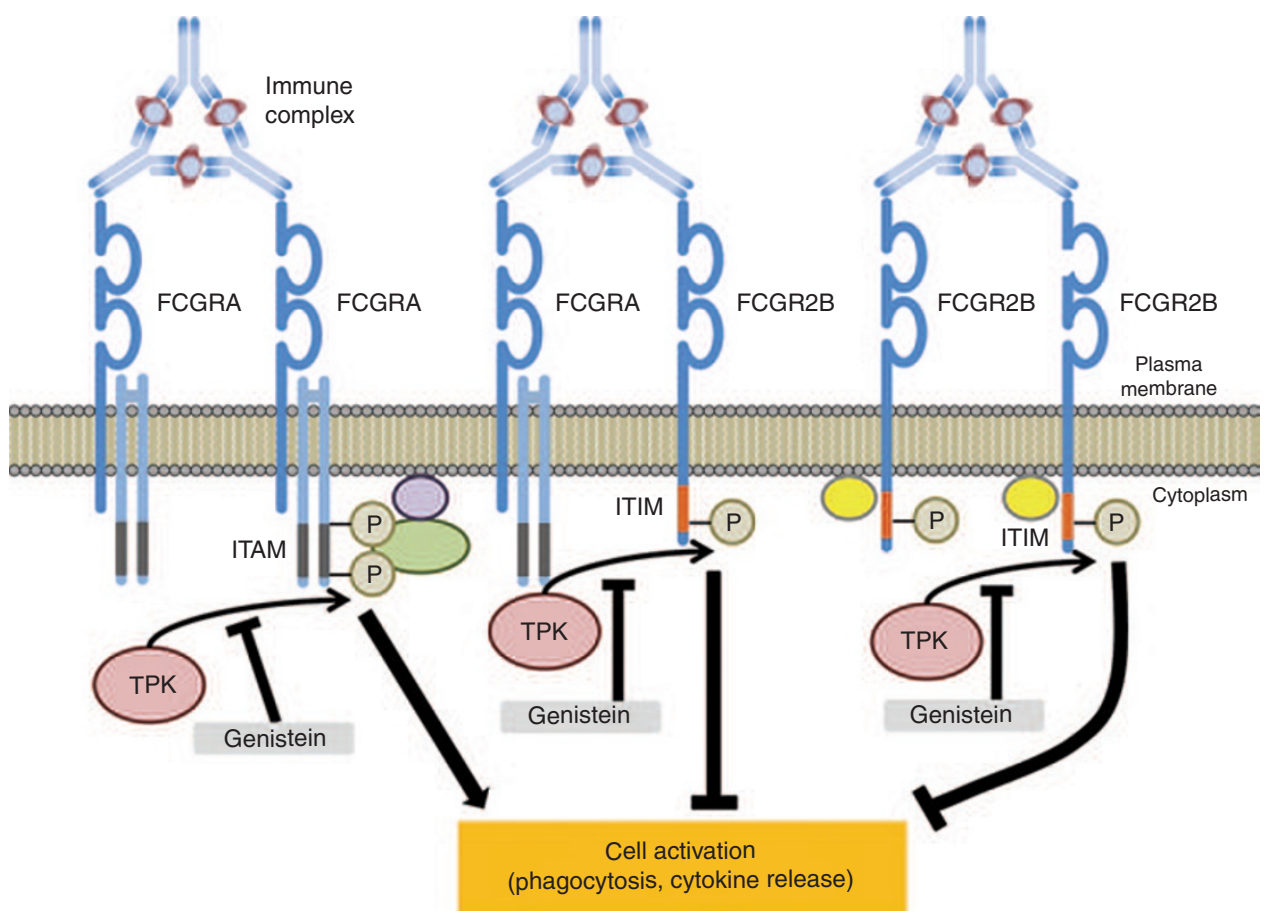

Figure 1. Schematic representation of potential interactions between an activating FCGR (FcGRA) and the inhibitory FcGR2B. Phosphorylation of immunoreceptor tyrosine-activating motifs (ITAMs) by tyrosine protein kinase (TPK) on cytosolic FcGRA tails or associated proteins (shown as violet and green, respectively) promotes cell activation. Similarly, phosphorylation of immunoreceptor tyrosine inhibitory motif (ITIM) on FCGR2B cross-linked with another FcGR and associated with signaling proteins (yellow) promotes cell inhibition. Genistein blocks phosphorylation by TPK, potentially disrupting the balance between activating and inhibiting signals. Modified with permission from ref. 15. 


\section{Integrated Mechanism Review}

vary among these ethnic groups. Therefore, the susceptibility conferred by FcGR2A131, although readily detectable, does not explain the racial/ethnic discrepancies in $\mathrm{KD}$ incidence.

The activating FcGR3B is expressed almost exclusively on neutrophils. Two FcGR3B single-nucleotide polymorphisms at positions 141 (rs403016) and 147 (rs447536) in the extracellular domain 1 lead to amino acid substitutions and result in two allotypic isoforms of the protein named neutrophil antigen, NA1 and NA2. A recent study identified the NA1 isotype as a major risk factor for IVIG refractoriness and development of persistent coronary artery dilation and/or aneurysm. NA1 shows selectively increased binding affinity for IgG1 and IgG3 as compared with NA2, suggesting that IVIG manipulates inflammation in KD through this neutrophilic receptor (12).

\section{TYROSINE PROTEIN KINASE INHIBITION AND ISOFLAVONES}

The consistent genetic data support the hypothesis that modifications in FcGR function or expression have an impact on KD susceptibility and/or IVIG treatment response. Genistein, the principal isoflavone in soy, also modifies the functions of these receptors by interfering with phosphorylation by tyrosine protein kinases $(14,21,22)$. The activating FcGRs either contain immunoreceptor tyrosine-activating motifs (ITAMs) in the cytoplasmic tail or associate closely with ITAM-containing cytosolic proteins (Figure 1). ITAM motifs are conserved four-amino-acid sequences in which tyrosine is separated from leucine by any two amino acids (-YxxL- (23)). Tyrosine protein kinases phosphorylate tyrosines within the ITAM following protein conformational changes induced by binding of immune complexes to the extracellular portion of the FcGR. ITAM phosphorylation permits docking of signaling proteins to the receptor molecule, which then initiates a cascade of events promoting inflammatory responses. The type of inflammatory response initiated varies according to the specific FcGR undergoing regulatory phosphorylation $(14,15)$ and whether the ITAM motif resides in the cytoplasmic tail of the FcGR or in an associated protein $(24,25)$. Tyrosine protein kinase also regulates the inhibitory FcGR2B signals via an immunoreceptor tyrosine-based inhibitory (ITIM) motif (Figure 2). Cross-linking of FcGR2B with activating FcGR receptors or B-cell receptors promotes tyrosine protein kinase-mediated ITIM phosphorylation, which leads to dephosphorylation and inhibition of the activating signal cascades $(15,26)$. Thus, the phosphorylation of ITIMs within FcGR2B plays a critical role in suppressing aberrant B-cell activation and potential autoimmunity. The overall effect of tyrosine protein kinase inhibition in humans in vivo is unknown. However, a selective tyrosine protein kinase inhibitor could potentially shift the balance in a manner that would prevent self-recognition and promote autoimmune inflammatory responses. The isoflavones genistein and daidzein are present chiefly in dietary soy (27). Genistein in soy occurs at nearly twice the concentration of daidzein and is used as a tyrosine protein kinase inhibitor in immunology laboratories. Genistein blocks phosphorylation by tyrosine protein kinases. The concentrations of genistein used to inhibit protein tyrosine kinases in vitro are generally

\section{Portman}

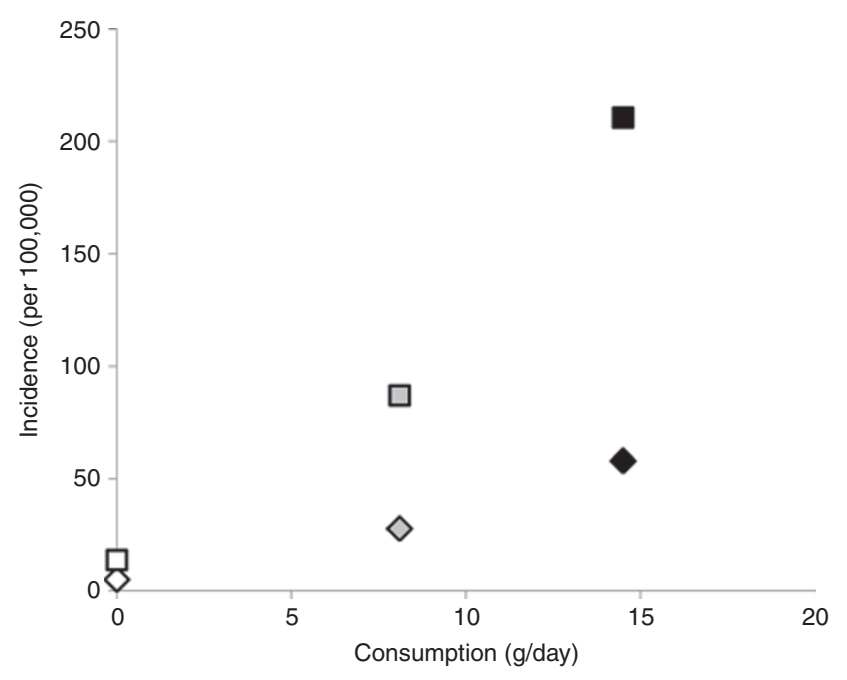

Figure 2. The relationship between Kawasaki disease (KD) incidence for children $<5$ y (boxes) or $<18$ y (diamonds) and adult soy consumption per day for three major ethnic groups in Hawaii (Caucasian in white, Native Hawaiian in gray, and Japanese in black). KD incidence is extracted from the work of Holman et al. (5), and soy consumption data are extracted from the work of Morimoto et al. (38). Symbols about the origin $(0,0)$ reflect low KD incidence and negligible soy consumption in Caucasians.

higher than are the plasma concentrations obtained through human oral ingestion of soy. However, immune-modulating effects, such as a marked reduction in thymic mass, have been observed in mice in vivo with plasma levels that approximate those obtained in humans by dietary ingestion (28). Specific inhibition of phosphorylating actions by protein tyrosine kinase on FcGRs or their associated ITAM-containing proteins occurs at fairly low concentrations in vitro (14). For instance, genistein inhibits phagocytosis, although not endocytosis, of immune complexes ligated to FcGR2A (14). Selective or differential inhibition by genistein according to FcGR isotype or ITAM vs. ITIM suggests that this isoflavone could disrupt the balance between activating and inhibitory signals. In addition, incomplete phosphorylation of ITAM tyrosines caused by genistein exposure may alter signal output and initiate an inhibitory signal (29).

Isoflavones have been implicated as susceptibility factors for autoimmune diseases in children. Fort et al. (30) showed increased frequency of soy-based feeding during infancy in children with autoimmune thyroiditis as compared with siblings and a healthy unrelated control group. The study was retrospective in nature and performed in a relatively small study population (31); however, the results have not been refuted.

\section{SOY CONSUMPTION AND KD INCIDENCE}

KD peakincidence occurs in children aged between 6 and $11 \mathrm{mo}$, and then slowly decreases with advancing age (2). Thus, prevailing theories regarding infectious etiology assume that $\mathrm{KD}$ results from an abnormal immune response to a first infection in predisposed individuals (32). The rarity of the disease over the first few months of life suggests that maternal passive immunity plays a protective role against the inciting 


\section{Kawasaki disease and isoflavones}

pathogen(s). Although maternal-fetal transmission of isoflavones is high (33), the earliest significant dietary exposure to isoflavones in children comes through either breast milk or soy-based infant formulas. Whereas ingestion of breast milk yields fairly low plasma isoflavone levels in nursing infants, soy formula-fed infants show substantially higher levels than those found in adults consuming soy (34), given that the formula is the infant's primary food. The estimated exposure rate to soy formula in American infants is similar to that in Japanese infants (10). Therefore, exposure over the first few months of age to isoflavones in soy-based formula does not account for the ethnic differences in KD incidence. However, Asian children beyond the age of conferred maternal immunity consume substantially more isoflavones than do Caucasian children of comparable age. Asian families typically add tofu to complement the infant breastfeeding or formula beginning at 4-6 mo (35). Tofu-fed infants show high isoflavone levels in plasma and urine, which exceed those found in Asian adults eating soy (34). The Japanese National Nutritional Survey (1995-2002) shows that by 1 year of age, Japanese children consume $~ 60 \%$ of the average daily soy and isoflavones ingested by adults between the ages of 20 and 40 y (36). Populations in other Asian countries vary substantially with regard to soy and isoflavone consumption. Furthermore, measures of consumption are based on different methodologies (36). Although the available data are highly variable, they consistently show that adult isoflavone consumption in Asian countries with substantially higher incidence rates for $\mathrm{KD}$ average between 11 and $40 \mathrm{mg}$ per day (36). By contrast, Caucasian populations in the United States and Western Europe with relatively low KD incidence consume $<2 \mathrm{mg}$ per day $(37,38)$. Thus, data by geographic region support the hypothesis that $\mathrm{KD}$ incidence is related to the amount of soy and isoflavone consumption.

The examination of published ethnic KD incidence rates in Hawaii removes any geographical influence in making these comparisons among ethnic populations. Japanese-American children in Hawaii display substantially higher incidence than do native Hawaiians, who in turn show higher rates than do Caucasian children (5). Although soy consumption in KD families in Hawaii has not been studied, the Multiethnic Cohort (Hawaii component) study has reported daily soy consumption in thousands of Caucasian, Native Hawaiian, and Japanese subjects (38). Data from the Multiethnic Cohort study published in 2010 were obtained for the same time period (1996-2004) as were the annual KD incidence data reported by Holman et al. (5) in 2011. These intriguing ethnicity data sets obtained from a defined geographic region, then combined, suggest an association between soy consumption and KD incidence (Figure 2) for all children (younger than $18 \mathrm{y}$ ) and for young children (aged younger than $5 \mathrm{y}$ ), when evaluated separately. The use of adult soy consumption represents a limitation for this analysis. Unfortunately, soy consumption in children by ethnic groups in the United States has not been studied. As noted, the data from Japan show that soy consumption in children is comparable to that in adults. Accordingly, one could assume that small children wean onto diets similar to those of their parents.

\section{Integrated Mechanism Review}

\section{CONCLUSION}

Genomic studies consistently show that the FcGRs play an important role in the pathogenesis of $\mathrm{KD}$ and in response to IVIG therapy. FcGR-regulated immune functions depend on initial ITAM or ITIM phosphorylation by tyrosine protein kinases. The isoflavone genistein inhibits action by these kinases, although the qualitative and quantitative responses vary by FcGR and immune cell type. Soy contains substantial quantities of isoflavones, and populations that historically consume large amounts of soy achieve readily measureable and fairly high levels of genistein in their plasma and of genistein metabolites in their urine. Populations with the highest isoflavone and soy consumption show the highest rates of KD. The presence of a moderating environmental factor fits well with the prevailing KD pathogenesis theories. Such a factor would promote susceptibility in infancy or early childhood after first exposure to an etiological agent. No study has previously considered diet as the important environmental component affecting risk for $\mathrm{KD}$, although other cardiovascular diseases have been linked to dietary agents. Well-designed epidemiological studies that include dietary history should be performed to determine if these isoflavones, and in particular genistein, affect risk for $\mathrm{KD}$.

\section{ACKNOWLEDGMENT}

The assistance of Masaki Kajimoto is appreciated for creating the figures.

\section{STATEMENT OF FINANCIAL SUPPORT}

This work was supported by a grant from the National Institutes of Health (Bethesda, MD; grant R21-HL90558) and a grant from the Thrasher Foundation, Salt Lake City, UT.

Disclosure: There is no conflict of interest.

\section{REFERENCES}

1. Kawasaki T, Kosaki F, Okawa S, Shigematsu I, Yanagawa H. A new infantile acute febrile mucocutaneous lymph node syndrome (MLNS) prevailing in Japan. Pediatrics 1974;54:271-6.

2. Nakamura Y, Yashiro M, Uehara R, et al. Epidemiologic features of Kawasaki disease in Japan: results of the 2009-2010 nationwide survey. J Epidemiol 2012;22:216-21.

3. Uehara R, Belay ED. Epidemiology of Kawasaki disease in Asia, Europe, and the United States. J Epidemiol 2012;22:79-85.

4. Salo E, Griffiths EP, Farstad T, et al. Incidence of Kawasaki disease in northern European countries. Pediatr Int 2012; e-pub ahead of print 21 June 2012.

5. Holman RC, Christensen KY, Belay ED, et al. Racial/ethnic differences in the incidence of Kawasaki syndrome among children in Hawaii. Hawaii Med J 2010;69:194-7.

6. Onouchi Y, Ozaki K, Burns JC, et al.; Japan Kawasaki Disease Genome Consortium; US Kawasaki Disease Genetics Consortium. A genome-wide association study identifies three new risk loci for Kawasaki disease. Nat Genet 2012;44:517-21.

7. Administration FaD. Food labeling health claims: final rule: soy protein and coronary heart disease. Fed Regist 1999;64;57700-33.

8. Xiao CW. Health effects of soy protein and isoflavones in humans. J Nutr 2008;138:1244S-9S.

9. Iqbal R, Anand S, Ounpuu S, et al.; INTERHEART Study Investigators Dietary patterns and the risk of acute myocardial infarction in 52 countries: results of the INTERHEART study. Circulation 2008;118:1929-37.

10. Bhatia J, Greer F; American Academy of Pediatrics Committee on Nutrition. Use of soy protein-based formulas in infant feeding. Pediatrics 2008;121:1062-8.

11. Khor CC, Davila S, Breunis WB, et al.; Hong Kong-Shanghai Kawasaki Disease Genetics Consortium; Korean Kawasaki Disease Genetics 


\section{Integrated Mechanism Review}

Consortium; Taiwan Kawasaki Disease Genetics Consortium; International Kawasaki Disease Genetics Consortium; US Kawasaki Disease Genetics Consortium; Blue Mountains Eye Study. Genome-wide association study identifies FCGR2A as a susceptibility locus for Kawasaki disease. Nat Genet 2011;43:1241-6.

12. Shrestha S, Wiener H, Shendre A, et al. Role of activating Fc $\gamma \mathrm{R}$ gene polymorphisms in Kawasaki disease susceptibility and intravenous immunoglobulin response. Circ Cardiovasc Genet 2012;5:309-16.

13. Shrestha S, Wiener HW, Olson AK, et al. Functional FCGR2B gene variants influence intravenous immunoglobulin response in patients with Kawasaki disease. J Allergy Clin Immunol 2011;128:677-80.

14. Huang ZY, Barreda DR, Worth RG, et al. Differential kinase requirements in human and mouse $\mathrm{Fc}$-gamma receptor phagocytosis and endocytosis. J Leukoc Biol 2006;80:1553-62.

15. Nimmerjahn F, Ravetch JV. Fcgamma receptors as regulators of immune responses. Nat Rev Immunol 2008;8:34-47.

16. Morris DL, Roberts AL, Witherden AS, et al. Evidence for both copy number and allelic (NA1/NA2) risk at the FCGR3B locus in systemic lupus erythematosus. Eur J Hum Genet 2010;18:1027-31.

17. Aloulou M, Ben Mkaddem S, Biarnes-Pelicot M, et al. IgG1 and IVIg induce inhibitory ITAM signaling through Fc $\gamma$ RIII controlling inflammatory responses. Blood 2012;119:3084-96.

18. Anthony RM, Ravetch JV. A novel role for the $\operatorname{IgG}$ Fc glycan: the anti-inflammatory activity of sialylated IgG Fcs. J Clin Immunol 2010;30: Suppl 1:S9-S14.

19. Anthony RM, Kobayashi T, Wermeling F, Ravetch JV. Intravenous gammaglobulin suppresses inflammation through a novel $\mathrm{T}(\mathrm{H}) 2$ pathway. Nature 2011;475:110-3.

20. Li X, Wu J, Carter RH, et al. A novel polymorphism in the Fcgamma receptor IIB (CD32B) transmembrane region alters receptor signaling. Arthritis Rheum 2003;48:3242-52.

21. Gaudette DC, Holub BJ. Effect of genistein, a tyrosine kinase inhibitor, on U46619-induced phosphoinositide phosphorylation in human platelets. Biochem Biophys Res Commun 1990;170:238-42.

22. Sargeant P, Farndale RW, Sage SO. The tyrosine kinase inhibitors methyl 2,5-dihydroxycinnamate and genistein reduce thrombin-evoked tyrosine phosphorylation and Ca2+ entry in human platelets. FEBS Lett 1993;315:242-6.

23. Turner M, Schweighoffer E, Colucci F, Di Santo JP, Tybulewicz VL. Tyrosine kinase SYK: essential functions for immunoreceptor signalling. Immunol Today 2000;21:148-54.

\section{Portman}

24. García-García E, Nieto-Castañeda G, Ruiz-Saldaña M, Mora N, Rosales C. FcgammaRIIA and FcgammaRIIIB mediate nuclear factor activation through separate signaling pathways in human neutrophils. J Immunol 2009;182:4547-56.

25. Rivas-Fuentes S, García-García E, Nieto-Castañeda G, Rosales C. Fcgamma receptors exhibit different phagocytosis potential in human neutrophils. Cell Immunol 2010;263:114-21.

26. Nimmerjahn F, Ravetch JV. Antibody-mediated modulation of immune responses. Immunol Rev 2010;236:265-75.

27. Cooke PS, Selvaraj V, Yellayi S. Genistein, estrogen receptors, and the acquired immune response. J Nutr 2006;136:704-8.

28. Cimafranca MA, Davila J, Ekman GC, et al. Acute and chronic effects of oral genistein administration in neonatal mice. Biol Reprod 2010;83:114-21.

29. Waterman PM, Cambier JC. The conundrum of inhibitory signaling by ITAM-containing immunoreceptors: potential molecular mechanisms. FEBS Lett 2010;584:4878-82.

30. Fort P, Moses N, Fasano M, Goldberg T, Lifshitz F. Breast and soy-formula feedings in early infancy and the prevalence of autoimmune thyroid disease in children. J Am Coll Nutr 1990;9:164-7.

31. Merritt RJ, Jenks BH. Safety of soy-based infant formulas containing isoflavones: the clinical evidence. J Nutr 2004;134:1220S-4S.

32. Pitzer VE, Burgner D, Viboud C, et al. Modelling seasonal variations in the age and incidence of Kawasaki disease to explore possible infectious aetiologies. Proc Biol Sci 2012;279:2736-43.

33. Adlercreutz H, Yamada T, Wähälä K, Watanabe S. Maternal and neonatal phytoestrogens in Japanese women during birth. Am J Obstet Gynecol 1999;180(3 Pt 1):737-43.

34. Franke AA, Halm BM, Custer LJ, Tatsumura Y, Hebshi S. Isoflavones in breastfed infants after mothers consume soy. Am J Clin Nutr 2006;84:406-13.

35. Quak SH, Tan SP. Use of soy-protein formulas and soyfood for feeding infants and children in Asia. Am J Clin Nutr 1998;68:Suppl 6:1444S-6S.

36. Messina M, Nagata C, Wu AH. Estimated Asian adult soy protein and isoflavone intakes. Nutr Cancer 2006;55:1-12.

37. Keinan-Boker L, Peeters PH, Mulligan AA, et al. Soy product consumption in 10 European countries: the European Prospective Investigation into Cancer and Nutrition (EPIC) study. Public Health Nutr 2002;5(6B): 1217-26.

38. Morimoto Y, Steinbrecher A, Kolonel LN, Maskarinec G. Soy consumption is not protective against diabetes in Hawaii: the Multiethnic Cohort. Eur J Clin Nutr 2011;65:279-82. 